\title{
HELIOSPHERIC MODULATION OF COSMIC RAYS: MODEL AND OBSERVATION
}

\author{
S.K. Gerasimova, P.Yu. Gololobov, V.G. Grigoryev, P.A. Krivoshapkin,

\section{G.F. Krymsky, S.A. Starodubtsev}

Yu.G. Shafer Institute of Cosmophysical Research and Aeronomy SB RAS, Yakutsk, Russia, s_k_gerasimova@ikfia.ysn.ru,gpeter@ikfia.sbras.ru,grig@ikfia.ysn.ru,p_a_krivoshapkin@mail.ru krymsky@ikfia.ysn.ru,starodub@ikfia.ysn.ru

This paper presents the basic model of cosmic ray modulation in the heliosphere, developed in Yu.G. Shafer Institute of Cosmophysical Research and Aeronomy of the Siberian Branch of the Russian Academy of Sciences. The model has only one free modulation parameter: the ratio of the regular magnetic field to the turbulent one. It may also be applied to the description of cosmic ray intensity variations in a wide energy range from $100 \mathrm{MeV}$ to 100 GeV. Possible mechanisms of generation of the turbulent field are considered. The primary assumption about the electrical neutrality of the heliosphere appears to be wrong, and the zero potential needed to match the model with observations in the solar equatorial plane can be achieved if the frontal point of the heliosphere, which is flowed around by interstellar gas, lies near the plane. We have revealed that the abnormal rise of cosmic ray intensity at the end of solar cycle 23 is related to the residual modulation produced by the subsonic solar wind behind the front of a standing shock wave. The model is used to describe features of cosmic ray intensity variations in several solar activity cycles.

Keywords Cosmic rays $\cdot$ Heliosphere $\cdot$ Modulation $\cdot$ Solar activity

\section{INTRODUCTION}

The solar activity (SA) cycle in cosmic ray (CR) intensity variations reflects the change in conditions of $\mathrm{CR}$ propagation in the heliosphere. The solar cycle is related to the interplanetary magnetic field (IMF) strength, solar wind (SW) velocity, geometry of regions of fast and slow SW, structure of the current sheet and its "corrugation" revealing itself as a sector structure of the magnetic field, and other parameters.

It is generally believed that CR penetrating into the heliosphere are affected by four main modulating processes:

1) convection caused by SW expansion;

2) diffusion due to particle scattering by turbulent IMF;

3) energy changes such as adiabatic energy losses and accelerations;

4) drift caused by the IMF gradient and curvature. 
These modulating processes are given by the CR transport equation [Krymsky, 1964; Parker, 1965]. The basic mathematical tools used for interpreting the observed CR modulation in the heliosphere are sketched in [Moraal, 2013]. The heliospheric modulation is expressed as an 11-year wave in CR intensity [Lockwood, Webber, 1967]. Long-term observations of this phenomenon show that cosmic rays differently behave during even and odd SA cycles; CR modulation has two peculiarities: under positive polarity of the Sun's general magnetic field, the CR intensity peak is flat; under negative polarity, it is sharp [Thomas et al, 2014.]. This causes the 22-year CR intensity variation, the so-called Hale cycle [Hale, Nicholson, 1925]. In [Charakhch'yan et al., 1973], such difference between SA cycles is attributed to the reversal of the Sun's general magnetic field. In [Levi, 1976; Jokipii et al., 1977; Jokipii, Thomas, 1981], this phenomenon is explained by the magnetic CR drift; then, the authors did numerous calculations of modulation with allowance made for a large number of parameters.

Modern models of galactic CR modulation in the heliosphere provide a correct and sufficiently detailed description of the processes, taking into account changes of all the above parameters in different combinations. As an example, we can mention works [Kota, Jokipii, 1983; Potgieter et al., 2001; Manuel et al., 2011; Manuel et al., 2014] and references therein. The theory considered in those and other works is based on the concept of CR diffusion and convection as well as on the concept of SW and magnetic drift.

A detailed description of this process requires computing three-dimensional models by numerical methods. Such programs have been developed by the above-mentioned authors. Kota and Jokipii in [Kota, Jokipii, 1983] study CR distribution with heliolongitude, heliolatitude, and distance from the Sun, as well as deformation of their energy spectrum. In [Potgieter et al., 2001; Manuel et al., 2011], the authors examine the CR effect of heliolatitude asymmetry at different polarities of the Sun's general magnetic field, as well as the time-dependent modulation. Laurenza et al. in [Laurenza et al., 2014] study the drift effect on CR modulation. Measurements of CR intensity (at the neutron monitor stations Climax, Rome, and Huancayo-Haleakala), area of sunspots, IMF magnitude, and tilt angle of the neutral current sheet by the method of empirical mode decomposition enabled the authors to assess the contribution of the drift effect on the GCR modulation. It is shown that depending on particle energy, the size of this contribution is up to 30-35\%. Moreover, it was found that this very effect is responsible for CR modulation during low SA. In particular, it is responsible for the flat (sharp) peak in even (odd) solar cycles. However, as the authors note, the contribution of drift effect decreases during periods close to maximum solar activity. Wibberenz et al. [Wibberenz et al., 2001] employ a simple model of propagating diffusion barrier to examine the influence of the tilt angle of the neutral current sheet and the heliospheric magnetic field magnitude on CR modulation.

Various researchers focus more on solar cycle 23, which was the longest; and the GCR flux at the end of the cycle reached its maximum value on record [Lockwood, 2010]. This minimum SA is considered abnormal. It offers a unique opportunity to test the theory of CR modulation, as well as to find true causes of such anomalous CR intensity. Gushchina et al. [Gushchina et al., 2014] tried to determine characteristics of CR intensity and to compare its variations in solar cycles 23-24 with those in cycles 19-22, using the previously developed [Gushchina et al., 2014] multiparameter model [Belov et al., 2001; Belov et al., 2002; Belov et al., 2007; Belov et al., 2005; Gushchina et al., 2008]. These authors have established that current cycle 24 is characterized by extremely weak CR modulation caused by anomalies of solar and heliospheric nature, in particular by reduction of the Sun's general magnetic field. Furthermore, they have found that the effect of the neutral current sheet tilt on CR modulation weakened in 20092012, although the tilt angle varied within the same ranges as in the previous cycles. 
Pacini, Usoskin [Pacini, Usoskin, 2015], by analyzing data from eight neutron monitors for SA cycle 24, have discovered that particles detected by polar neutron monitors experience a weaker heliospheric modulation during the last cycle than in the previous cycles. At the same time, higher energy particles are free from such significant changes during modulation. The authors attribute this phenomenon to a decrease in the level of IMF turbulence during the last solar cycle [Bisoi et al., 2014; Starodubtsev, Grigoryev, 2011]. The turbulence significantly affects CR propagation in the heliosphere. A similar conclusion, but through numerical simulation of CR transport equation and neutron monitor data, has been drawn in [Zhao et al., 2014].

Ferreira, Potgieter [Ferreira, Potgieter, 2004] have developed a multiparameter (compound) model which enables determination of time dependences of coefficients in the CR transport equation from experimental data. Then, the model [Manuel et al., 2014] satisfactorily described the behavior of CR intensity recorded by spacecraft Voyager-1 and 2, IMP-8, and Ulysses. According to conclusions made by the above authors, the observed behavior of CR intensity in 2004-2010 is primarily due to changes in the diffusion coefficient rather than in the drift coefficient.

The advantage of all the above works is that they take into account the multiple factors that vary with solar cycle, and describe certain details of CR modulation in the heliosphere. However, such a detailed description may also be perceived as a disadvantage: in these calculations, it is difficult to find the main factor and to trace the dominant modulation process. We think that the opposite formulation of the problem is possible: elimination of unnecessary details from the heliospheric model, its maximum simplification, and determination of the only factor that varies with solar cycle and significantly affects the long-term CR modulation.

In this paper, we discuss the results of calculations in this very problem setting.

\section{HELIOSPHERIC MODEL AND SOLAR CYCLE}

The model heliosphere is a region bounded by a radius $\approx 100$ A.U., beyond which there is steady CR intensity such as in the interstellar medium. The radius has very little effect on the results obtained. The heliosphere is filled by a uniform radial SW having velocity of $400 \mathrm{~km} / \mathrm{s}$, not varying with the solar cycle. The radial component of the regular magnetic field does not depend in magnitude on heliolatitude and has opposite signs in the northern and southern hemispheres. The current sheet separating the hemispheres is located in the solar equatorial plane and has no corrugation. The geometry of the regular field is completely determined by the kinematics combining SW with the solid-body rotation of the Sun. The regular field is superimposed by the turbulent one. The ratio of intensities of these two fields is the same throughout the heliosphere, but varies with solar cycle. For 4.5 years, the turbulent field increases linearly with time from minimum SA, where it represents a small fraction of the regular field, to maximum SA, where the regular field becomes vanishingly weak. Then, the turbulent field linearly decreases for 6.5 years. The total field does not change with SA cycle. In fact, it is not so, but changes in the total field are much smaller than those in either of these two fields. The regular component changes its sign at maximum SA.

\section{TRANSPORT EQUATION. TRANSPORT COEFFICIENTS}

The solar wind is magnetized, and CR in it are transported along with the magnetic field. They are also scattered by magnetic irregularities, thus being diffused. In the simplest model of Bohm diffusion, they are scattered with the time constant $\tau=\omega_{\mathrm{T}}^{-1}$, where $\omega_{\mathrm{T}}=e H_{\mathrm{T}} v / p c, e$ is the electron charge, $H_{\mathrm{T}}$ is the turbulent field strength, $c$ is the 
velocity of light, $p, v$ are the momentum and velocity of particles. Diffusion in the real IMF differs from Bohm diffusion in terms of the presence of the regular field component $H$, hence diffusion becomes anisotropic. The simplest model of this process in case of instant scatterings is described by the diffusion tensor

$$
\kappa_{\alpha \beta}=\kappa_{\|} h_{\alpha} h_{\beta}+\kappa_{\perp}\left(\delta_{\alpha \beta}-h_{\alpha} h_{\beta}\right)+\kappa_{\mathrm{H}} h_{\gamma} e_{\alpha \beta \gamma},
$$

such that

$$
\kappa_{\|}=v^{2} \tau / 3 ; \kappa_{\perp}=\kappa_{\|} /\left(1+k^{2}\right) ; \kappa_{\mathrm{H}}=\kappa_{\|} k /\left(1+k^{2}\right) ; k=\omega \tau,
$$

$h_{\alpha \beta \gamma}$ are components of the unit vector of the regular magnetic field, $\delta_{\alpha \beta}$ is the unit tensor, $e_{\alpha \beta} \gamma$ is the absolutely antisymmetric unit tensor, $v$ is the particle velocity, $\tau$ is the average time between scattering events, $\omega$ is the gyrofrequency of CR particles in the regular magnetic field.

The parameter $k=\omega \tau$ (it may be called a degree of regularity of the magnetic field) is a key parameter for describing the modulation process. The first simplification concerns the behavior of this parameter: it will be considered constant for the entire heliosphere and independent of particle energy, even if it varies with SA cycle. Note that the single value of $k$ for different energy ranges indicates a steady shape of the spectrum of the SW magnetic turbulence, which in the model is accepted as a power one with an exponent -1 . This is in illusory contradiction with direct measurements of IMF fluctuation spectra [Starodubtsev, Grigoryev, 2011]. However, it should be taken into account that $\mathrm{CR}$ reflect the character of the turbulence throughout the heliosphere, which, of course, differs from the turbulence near Earth's orbit.

The antisymmetric part of the diffusion tensor, which is provided by the vector product and describes the socalled Hall diffusion, can be taken as a separate term. The term of the transport equation containing the Hall diffusion can be represented as $-\vec{u}_{\mathrm{dr}} \operatorname{grad} f$, where $f$ is the particle distribution function that depends on spatial coordinates and particle momenta and

$$
-\vec{u}_{\mathrm{dr}}=\frac{\mathrm{v}}{3} \frac{k^{2}}{k^{2}+1} \frac{p c}{e} \operatorname{rot} \frac{\vec{H}}{H^{2}}
$$

is the drift velocity.

The equation of particle transport by the solar wind with a velocity $u$ in a drift form contains only a symmetrical diffusion tensor, which we denote $\tilde{\kappa}$ :

$$
\frac{\partial f}{\partial t}=\operatorname{div}(\tilde{\kappa} \operatorname{grad} f)-\left(\vec{u}+\vec{u}_{\mathrm{dr}}\right) \operatorname{grad} f+\frac{1}{3} \operatorname{div} \vec{u} p \frac{\partial f}{\partial p} .
$$

Such a form of equation with an isolated drift term was probably used for the first time in [Jokipii et al., 1977].

Let us make some comments about the drift velocity $\vec{u}_{\mathrm{dr}}$, that reflects the drift of particles in an inhomogeneous magnetic field. In the presence of turbulence, the drift velocity decreases, and only if $k>>1$ it coincides with the velocity of the real drift. When the magnetic field changes sign, the direction of particle drift reverses, and this seems very important because IMF periodically reverses its polarity. 
With a sufficient accuracy, the GCR modulation can be considered in the approximation which is linear in the solar wind velocity (see [Krymsky et al., 2007]). Assuming that the solar wind velocity is a small parameter, we represent the distribution function as a sum of variable and invariable parts. Denote the invariable part by $f_{0}$ and the variable part by $f$. In the stationary case, the linearization of transport equation (1) in $\vec{u}$ yields

$$
\nabla(\tilde{\kappa} \nabla f)-\vec{u}_{\mathrm{dr}} \nabla f=\frac{2(\gamma+2)}{3} \frac{u_{0}}{r} f_{0} .
$$

The term on the right-hand side is defined by SW velocity divergence equal to $2 u_{0} / r$, and the $f_{0}$ dependence on the momentum is represented by a power function with an exponent equal to $-(\gamma+2)$.

\section{TRANSPORT EQUATION IN THE INTERPLANETARY MAGNETIC FIELD}

The Sun's general magnetic field frozen in SW plasma is carried out to large distances like the interplanetary field. Since the radial component of the dipole field becomes zero in the equatorial plane, there is a heliolatitudinal magnetic pressure gradient diverting the radial flow to the equatorial plane so that the radial field strength would be uniformly distributed over the heliolatitude. The calculations and observations demonstrate that the homogeneous magnetic field distribution is a good approximation.

The solar rotation with an angular velocity $\omega_{\odot}$ gives rise to the azimuthal field component.

The magnetic field in the whole region of the supersonic wind is represented by

$$
\begin{gathered}
H_{r}=H_{0} \frac{r_{0}^{2}}{r^{2}} \operatorname{sign}\left(\frac{\pi}{2}-\theta\right), \\
H_{\varphi}=-H_{0} \frac{r_{0}}{r} \sin \theta \operatorname{sign}\left(\frac{\pi}{2}-\theta\right) .
\end{gathered}
$$

As the length scale $r_{0}$ it is convenient to choose the ratio $u_{0} / \omega_{\odot}$, then at $r_{0}$ the azimuthal field component near the equatorial plane and the radial component are equal (designated as $H_{0}$ ). Given an SW velocity $u_{0}=400 \mathrm{~km} / \mathrm{s}, r_{0}$ is approximately equal to 1 A.U. The polar angle $\theta$ is measured from the axis of rotation to the south.

It is assumed that the dipole magnetic moment and the Sun's rotational moment are parallel, therefore the radial field component is positive in the northern hemisphere $(\theta<\pi / 2)$ and negative in the southern hemisphere. Every 11 years during maximum of the subsequent solar cycle, the magnetic field reverses polarity, and hence we have to change the magnetic field sign in calculation formulas.

In most part of the heliosphere, the radial magnetic field can be regarded as negligible. The radial component is held only if $r<(2 \div 3) r_{0}$. The large-scale modulation pattern will, of course, be determined by the "far-field" zone, where the field is purely azimuthal. 
Diffusion and drift velocity coefficients for the far-field zone are represented by

$$
\begin{aligned}
& \kappa_{r r}=\kappa_{\theta \theta}=\kappa_{\perp}=\frac{v r_{0}}{3} \frac{p}{p_{0}} \frac{k}{k^{2}+1} \frac{r}{r_{0} \sin \theta}, \\
& u_{r}=v \frac{p}{p_{1}} \delta\left(\theta-\frac{\pi}{2}\right), u_{\theta}=v \frac{p}{p_{1}} \frac{\operatorname{sign}\left(\frac{\pi}{2}-\theta\right)}{\sin \theta} .
\end{aligned}
$$

Here

$$
p_{1}=\frac{3}{2} \frac{k^{2}+1}{k^{2}} p_{0} ; p_{0}=\frac{e H_{0} r_{0}}{c} .
$$

The delta function derives from the presence of the current sheet in the equatorial plane and appears at the formal differentiation of discontinuous multiplier. We can obtain the characteristic scale for $p_{0}$ if the SW velocity is taken to be $400 \mathrm{~km} / \mathrm{s}$ and the magnetic field strength in Earth's orbit is $\sim 5 \mathrm{nT}$. With these parameters $r_{0}=R_{\oplus}=1.5 \cdot 10^{13}$ $\mathrm{cm} H_{0}=3 \cdot 10^{-5} \mathrm{G}$, and $p_{0} \approx 150 \mathrm{GeV} / \mathrm{s}$. If $k^{2}>>1$, then for the energy of $\sim 15 \mathrm{GeV}$, which is close to the effective energy of particles detected by neutron monitors, a typical value of drift velocity is $2 \cdot 10^{4} \mathrm{~km} / \mathrm{s}$, which is almost two orders of magnitude greater than the SW velocity. As the particle energy increases, this value will be even greater.

Note that the diffusion coefficient and the drift velocity become infinite on the symmetry axis. Physically, this is due to the fact that the magnetic field strength is extremely low here. In the approximation we adopt, it formally vanishes.

The transport equation can be put in a convenient form if we introduce the heliolatitude $\psi=\pi / 2-\theta$ and variable $\lambda=-\ln (r / R)$, where $R$ is the radius of the heliosphere:

$$
\frac{1}{2 k} \Delta f-\frac{1}{k} \frac{\partial f}{\partial \lambda}+\frac{\partial f}{\partial \lambda} \delta(\psi)+\frac{\partial f}{\partial|\psi|}=b_{1} \cos \psi
$$

Here

$$
b_{1}=\frac{2(\gamma+2)}{3} \frac{u_{0}}{v} \frac{p_{1}}{p} f_{0},
$$

and the Laplace operator acts on the variables $\lambda, \psi$ as on Cartesian coordinates.

\section{HELIOPAUSE}

The supersonic wind has a dynamic pressure that decreases quadratically with distance due to radial expansion. In Earth's orbit, typical wind parameters are as follows: particle density $n_{0}=8 \mathrm{~cm}^{-3}$, velocity $u_{0}=400 \mathrm{~km} / \mathrm{s}$. Since the main composition includes protons $\left(1.7 \cdot 10^{-24} \mathrm{~g}\right)$, the typical dynamic wind pressure $p u^{2}=2 \cdot 10^{-8} \mathrm{dyne} / \mathrm{cm}^{2}$.

The density of CR energy and other components of the interstellar medium is by order of magnitude equal to 1 $\mathrm{eV} / \mathrm{cm}^{3}$. This corresponds to a pressure of $\sim 10^{-12} \mathrm{dyne} / \mathrm{cm}^{2}$. Thus, at a distance of about 100 A.U. SW should under- 
go a shock transition and become subsonic. We can assume approximately that the modulating effect of SW at this distance is terminated. The said boundary of the supersonic wind is called the heliopause.

The CR modulation in the heliosphere depends on conditions at the heliopause. At present, in the absence of precise knowledge about the heliopause, boundary conditions have to be formulated with the use of model representations. The presence of a large-scale electric field in the heliosphere testifies that different heliospheric regions have different potentials with respect to the external medium.

Note that the question concerning the GCR modulation effect of the potential difference between different boundary points and outer heliospheric regions has been simultaneously and independently examined by different researchers (see, e.g., [Jokipii, Levy, 1979; Krajnev, 1979, Krajnev, Kalinin, 2003]).

Considering the heliopause in the first approximation as a heliosphere boundary, we conclude that there is a jump of potential at it.

$$
\Delta U=-\frac{u H_{0} r_{0}}{c}(\sin |\psi|+C)
$$

If we assume that the heliosphere as a whole is neutral, then $C=-1 / 2$.

The above SW parameters give a heliospheric potential equal to $\pm 100 \mathrm{MV}$ in the equatorial plane and a potential of the same magnitude but with opposite sign at the poles.

For the positive polarity of the magnetic field, the potential of the low-latitude heliosphere is positive. In this case, for brevity we call the heliosphere positively charged. The magnetic field reversal causes the potentials to change sign.

The CR modulation by an electrostatic field can be calculated by application of the Liouville equation, which in our case takes the form

$$
\cup \frac{\partial f}{\partial \vec{r}}+e \vec{E} \frac{\partial f}{\partial p}=0
$$

Since $\vec{E}=-\nabla U$, and $\mathrm{v}=\partial \varepsilon / \partial p$, the total integral of this equation depends only on the particle energy $\varepsilon$ :

$$
f_{0}(p)+f(\vec{r}, p)=F(\varepsilon+e U) .
$$

Assuming that the distribution function outside the modulation region is equal to

$$
f_{0}=B p^{-(\gamma+2)}
$$

we get

$$
\begin{aligned}
& f=B\left[\left(\frac{\varepsilon+e U}{c}\right)^{2}-(m c)^{2}\right]^{-\frac{(\gamma+2)}{2}}-B p^{-(\gamma+2)} \approx \\
& \approx-(\gamma+2) \frac{e U \varepsilon}{(p c)^{2}} f_{0} .
\end{aligned}
$$


The last approximate equality is valid if $p>>e U / c$. Accordingly, for example, at an energy of $10 \mathrm{GeV}$ the electrostatic modulation is about $\pm 5 \%$ and should be taken into account in calculations.

\section{BOUNDARY CONDITIONS}

If we claim that the distribution function depends on the modulus of the heliolatitude, we obtain for the second derivative

$$
\frac{\partial^{2} f}{\partial \psi^{2}}=\frac{\partial}{\partial \psi}\left(\frac{\partial f}{\partial|\psi|} \operatorname{sign} \psi\right)=\frac{\partial^{2} f}{\partial|\psi|^{2}}+2 \frac{\partial f}{\partial|\psi|} \delta(\psi)
$$

Omitting the sign of the $\psi$ modulus in $0 \leq \psi \leq \pi / 2$, we derive a modulation equation for the far-field zone instead of (6):

$$
\frac{1}{2 k} \Delta f-\frac{1}{k} \frac{\partial f}{\partial \lambda}+\frac{\partial f}{\partial \psi}=b_{1} \cos \psi
$$

and at $\psi=0$ we have a boundary condition

$$
\frac{1}{k} \frac{\partial f}{\partial \psi}+\frac{\partial f}{\partial \lambda}=0
$$

arising from the need for mutual offset of singular terms.

The boundary condition at the heliopause $(\lambda=0)$ is determined with respect to the electric potential $\Delta \mathbf{U}$ :

$$
f=b_{1} \frac{k^{2}}{k^{2}+1}(-1 / 2+\sin \psi) \text {. }
$$

Here, $f$ is the variable part, and the full description requires us to add the undisturbed part $f_{0}$.

In this boundary condition, the amplitude dependent on the IMF strength, SW velocity, and particle momentum is for convenience expressed through the same, above introduced parameter $b_{1}$. With $\psi=\pi / 2$, because of the infinite quantity of the diffusion function, the distribution coefficient is constant and equal to

$$
\left.f\right|_{\psi=\pi / 2}=\frac{b_{1}}{2} \frac{k^{2}}{k^{2}+1}
$$

Thus, equation (8) and three boundary conditions (9-11) determine the behavior of the function $f$ in the range $\lambda \geq 0,0 \leq \psi \leq \pi / 2$. The equation and boundary conditions in passing to the negatively charged heliosphere are modified by changing simultaneously the sign of $k$ and $p_{0}$ (and thus of $p_{1}$ and $b_{1}$ ).

If the calculations are aimed at finding the modulation near Earth's orbit, we have a point $(\lambda \approx 4.6$; $\psi=0)$ in the vicinity of which we need to define the behavior of the distribution function. This point is by respectively 4.6 and $\pi / 2$ dimensionless units apart from the boundaries of the region. These distances are of the same order of magnitude, and the influence of any of these boundaries crucially depends on the amount and direction of particle drift. 
In the positive heliosphere, the drift is directed toward the equatorial plane and along it outward, therefore the main influence is exerted by the high-latitude boundary $\psi=\pi / 2$. With the negative polarity, the opposite direction of the particle drift surpasses the effect of the high-altitude boundary, and proper allowance must be made for conditions at the heliopause. Only at $k<1$ when the drift is small, the high-latitude boundary contributes to the solution for the negative heliosphere.

\section{POSITIVE AND NEGATIVE LOW-LATITUDE HELIOSPHERE}

For the positive heliosphere, neglecting the heliopause effect, we find the solution to $f_{\mathrm{g}}(\psi)$ independent of $\lambda$ by two successive integrations of linear equation (8) with respect to $\psi$; the integration constants are determined from the conditions at $\psi=0$ and $\psi=\pi / 2$ :

$$
\begin{aligned}
& f_{g}(\psi)=\frac{2 k b_{1}}{4 k^{2}+1}(2 k \sin \psi-\cos \psi-2 k)+ \\
& +\frac{2 k b_{1}}{4 k^{2}+1}\left(e^{-2 k \psi}-e^{-k \pi}\right)+\frac{b_{1}}{2} \frac{k^{2}}{k^{2}+1}
\end{aligned}
$$

In the equatorial plane, this function is

$$
f_{g}^{+}(0)=-\frac{4 k^{2} b_{1}}{4 k^{2}+1}-\frac{2 k b_{1}}{4 k^{2}+1} e^{-k \pi}+\frac{b_{1}}{2} \frac{k^{2}}{k^{2}+1} .
$$

For negative polarity, the $\lambda$-independent solution also exists formally; it is obtained by changing the sign of $k$ and $b_{1}$ and for the equatorial plane takes the form

$$
f_{g}^{-}(0)=\frac{4 k^{2} b_{1}}{4 k^{2}+1}-\frac{2 k b_{1}}{4 k^{2}+1} e^{k \pi}-\frac{b_{1}}{2} \frac{k^{2}}{k^{2}+1}
$$

The modulation depth is seen to be very great. As already mentioned, the reason for this is the drift toward both sides from the equatorial plane, so it is necessary to take into account the heliopause effect. If we restrict ourselves to not too small $k$, we can ignore the influence of the high-latitude boundary and consider the behavior of the function at low heliolatitudes, where $\sin \psi \approx \psi, \cos \psi \approx 1$.

The equation (here $k>0, b_{1}>0$, the sign of $\partial f / \partial \psi$ changed)

$$
\frac{1}{2 k} \Delta f-\frac{1}{k} \frac{\partial f}{\partial \lambda}-\frac{\partial f}{\partial \psi}=b_{1}
$$

with boundary conditions

$$
\begin{aligned}
& \frac{1}{k} \frac{\partial f}{\partial \psi}-\frac{\partial f}{\partial \lambda}=\left.0\right|_{\psi=0}, \\
& f=\left.b_{1} \frac{k^{2}}{k^{2}+1}(1 / 2-\psi)\right|_{\lambda=0}
\end{aligned}
$$

has a simple solution in this case 


$$
f=f_{l}=b_{1} \frac{k^{2}}{k^{2}+1}\left(1 / 2-|\psi|-\frac{\lambda}{k}\right)
$$

Here, the sign of the $\psi$ modulus is restored. Note that the function $f$ is symmetric about the equatorial plane, and its derivative with respect to $\psi$ has a discontinuity at $\psi=0$ so that the behavior of $f$ has a "wedge-shaped" character.

\section{22-YEAR CYCLE}

The reversal of the Sun's general magnetic field and consequently of IMF during periods of maximum SA results in mismatch between CR modulation characters in two consecutive 11-year cycles. The full CR modulation cycle is therefore the 22-year cycle.

The CR modulation for the positive polarity is described by formula (12); and for the negative one, formula (14) should be applied.

At a high turbulence level (with $k^{2}<<1$ ), we should consider both channels of particles: along the equatorial plane (solution (14)) and from high latitudes (solutions (12) and (13)). In the general case, with a reasonable degree of accuracy their joint action can be described by the formula

$$
f(k)=\frac{f_{l}(0) f_{g}(0)}{f_{l}(0)+f_{g}(0)}
$$

For further consideration, it is convenient to enter the modulation function $F(k)$ depending only on $k$ :

$$
F(k)=f / b_{1} .
$$

To draw a theoretical picture of the modulation, we maximally simplify the assumption about the relation between the parameter $k$ and the phase of the 11-year cycle. For the phase of a cycle $\phi$ we take a value varying from 0 during minimum SA to 1 during maximum SA and back from maximum to minimum. The phase, by definition, changes linearly with time at each of the said time intervals. The magnetic field reversal occurs at cycle maximum when $\phi=1$. The period of SA increase is set equal to 4.5 years; and the period of SA decrease, to 6.5 years.

$$
k=\omega \tau=H_{0} / H_{\mathrm{T}} .
$$

Assume that the turbulent field strength reaches its maximum at the moment of reversal and linearly depends on the phase of a cycle:

$$
H_{\mathrm{T}}=H_{\text {obs }}\left(\phi+1 / k_{0}\right) .
$$

The small increment $1 / k_{0}$ determines the residual level of turbulence during minimum SA. We consider the observed field $H_{\mathrm{obs}}$, which is the sum of regular and turbulent fields, to be constant throughout the cycle. The real field undergoes changes in the cycle, but they are considerably smaller than those characterizing the regular and turbulent field separately. Hence, 


$$
H_{\text {набл }}=\sqrt{H_{0}^{2}+H_{\mathrm{T}}^{2}}=\text { const } .
$$

From this it follows that the regular field during maximum SA is much smaller than the turbulent one.

As a result, the parameter $k$ depends on the phase as follows:

$$
k=\frac{\sqrt{1-\phi^{2}}}{\phi+1 / k_{0}}
$$

and varies within $0<k<k_{0}$.

In accordance with the above assumptions, the field strength $H_{0}$ in expressions (5) and (6) for $p_{0}, p_{1}$, and $b_{1}$ becomes time-dependent, and the constant $b$ should be multiplied by an additional correction factor

$$
\eta=\sqrt{1-\phi^{2}}
$$

\section{EXTENSION OF THE APPLICABILITY \\ OF THE MODEL INTO THE LOW-ENERGY REGION}

The core theory of heliospheric modulation uses a linear wind-velocity approximation and therefore is suitable only for describing high-energy CR variations. Let us try to generalize this theory to a lower-energy region, and then compare with the results of multi-year CR observations in the stratosphere, made by an LPI group in Moscow and Murmansk (reported in [Stozhkov et al., 2009]), and with satellite observations.

In the CR transport equation,

$$
\nabla(\kappa \nabla f)-\left(\vec{u}+\vec{u}_{d r}\right) \nabla f+\frac{1}{3} \nabla \vec{u} p \frac{\partial f}{\partial p}=0
$$

the distribution function is represented as $f(p, \vec{r})=f_{0}(p) \exp (-\zeta(p, \vec{r}))$, where $f_{0}(p)$ is the undisturbed function depending only on $p$ and having a power form, and $\zeta$ is the modulation-induced disturbance. Then, upon the substitution we obtain

$$
\begin{aligned}
& -\nabla(\kappa \nabla \zeta)+\kappa(\nabla \zeta)^{2}+\left(\vec{u}+\vec{u}_{\mathrm{dr}}\right) \nabla \zeta+ \\
& +\frac{1}{3} \nabla \vec{u}\left(\frac{p}{f_{0}} \frac{\partial f_{0}}{\partial p}-p \frac{\partial \zeta}{\partial p}\right)=0 .
\end{aligned}
$$

Assuming that the squared gradient $\zeta$ is small, and the $\zeta(p)$ dependence is weak compared to $f_{0}(p)$, we omit the respective terms. The resulting equation for $\zeta$ is no different from the previously examined linear equation and provides us with the necessary solution through $F(k)$ (see (15)).

$$
\zeta=F(k) b_{1} / f_{0}
$$


To compare the solution with the CR observations in the stratosphere [Stozhkov et al., 2009], we should average it over particle momenta. Since the dependence of the solution on the momentum now becomes more complicated, the averaging over the entire primary spectrum should be carried out explicitly. To do this requires knowing the generation multiplicity of secondary particles $m(p)$, which represents a contribution of CR with $p$ to the observed intensity calculated for one primary particle. For it the following expression is used: $m(p)=\operatorname{const}\left(1-p_{m}^{2} / p^{2}\right) p$, where $p_{m}=1.2 \mathrm{GeV} / \mathrm{s}$ is the ionization cutoff of the multiplicity for vertically incident particles at a depth of 100 $\mathrm{g} / \mathrm{cm}^{2}$. This dependence follows from the assumption that the multiplication of a primary particle in the atmosphere at the depth of observation (at the intensity maximum) is completed. The expression in brackets suggests that at low energies due to ionization cutoff at the maximum intensity there are particles moving only within their energydependent solid angle near the vertical.

If we now go to even lower-energy particles $(100 \mathrm{MeV})$, the term $(1 / 3) \nabla \vec{u} p \partial \zeta / \partial p$ cannot be neglected any more as it changes sign in the CR spectrum.

Consequently, the solution $\zeta(p)$, obtained in the linear approximation, is to be multiplied by the correction factor; then it takes the form

$$
\zeta(p)=b_{0} F(k)\left(1-\frac{p}{\gamma+2} \frac{\partial \zeta}{\partial p}\right)
$$

Here, $b_{0}$ differs from $b_{1}$ only in respect to the absence of $f_{0}$ and can be expressed as $b_{0}=\sigma_{0} / p^{2}$, where

$$
\sigma_{0}=(\gamma+2) m_{\mathrm{p}} u e H_{0} r_{0} / c
$$

and $m_{\mathrm{p}}$ is the proton mass. Substituting numbers yields $\sigma_{0}=0.9(\mathrm{GeV} / \mathrm{s})^{2}$. Adding the notation $-F(k) \sigma_{0} /(\gamma+2)=g$, we derive the linear differential equation

$$
\zeta^{\prime}-\frac{p}{g} \zeta-\frac{\gamma+2}{p}=0
$$

whose solution is

$$
\zeta=-\frac{\gamma+2}{2} \exp \left(p^{2} / 2 g\right) E_{1}\left(p^{2} / 2 g\right)
$$

and $E_{1}(x)=\int_{x}^{\infty}(\exp (-x) / x) d x$ is the exponential integral. It must be understood that in the said energy range $\gamma \approx 0$.

Thus, with $\zeta$, we can now write the solution to the problem of heliospheric modulation of low-energy particles. Only one comment should be made: at low diffusivity, our quasistationary solution needs to be corrected. The diffusion scale at $\kappa \sim r$, as in our case, increases linearly with time. For $270 \mathrm{MeV}$ protons, it reaches 100 A.U. for about 1 year. This is the period of diffusion filling of the heliosphere. The convection displacement of CR from the heliosphere takes approximately the same time. Therefore, calculation data should be smoothed with an interval of 1 year. 


\section{COMPARING MODEL PREDICTIONS WITH OBSERVATIONS}

The calculation of modulation for $13 \mathrm{GeV}$ particles, made under the above simplified conditions for $k_{0}$ equal to 3, 5, and 10, is illustrated in Figure 1. Earth was assumed to be in the solar equatorial plane. For convenience, we present the modulation pattern for two successive 22-year cycles. The same figure shows the intensity of the neutron CR component. The data have been taken from [Jokipii, Kota, 1997]. They were acquired from CR intensity observations made on Mount Climax for almost 50 years. It is apparent that the 11-year cycles of modulation differ significantly. The cycles with the polarity reversal from "+" to “-” (odd SA cycles) have a longer period of CR intensity decrease (broad minimum), whereas the recovery of their intensity takes less time (sharp peak) than in subsequent cycles.

The broad intensity minimum stems from the fact that after the reversal the CR intensity continues to decrease, although the turbulence level has begun going down. The reason for this delay is the CR drift from the equator to high latitudes under negative polarity of the Sun's general magnetic field. This drift impedes the arrival of cosmic rays from high latitudes. It occurs after maximum solar activity when $k$ becomes sufficiently large.

With further decrease in the turbulence level (with increasing $k$ ), CR drifting in the equatorial plane of the heliosphere boundary begin to make their own contribution. This contribution, as it increases, leads to recovery of the intensity.

The "flat" intensity maximum under positive polarity of the heliosphere is also associated with the drift which in this case is directed from high latitudes to the equator.

If the level of residual turbulence during minimum SA is not too high $\left(k_{0} \gtrsim 10\right)$, the maximum intensity (sharp peak) is higher than that of CR without modulation. This is due to the action of the heliospheric electric field, as already mentioned above.

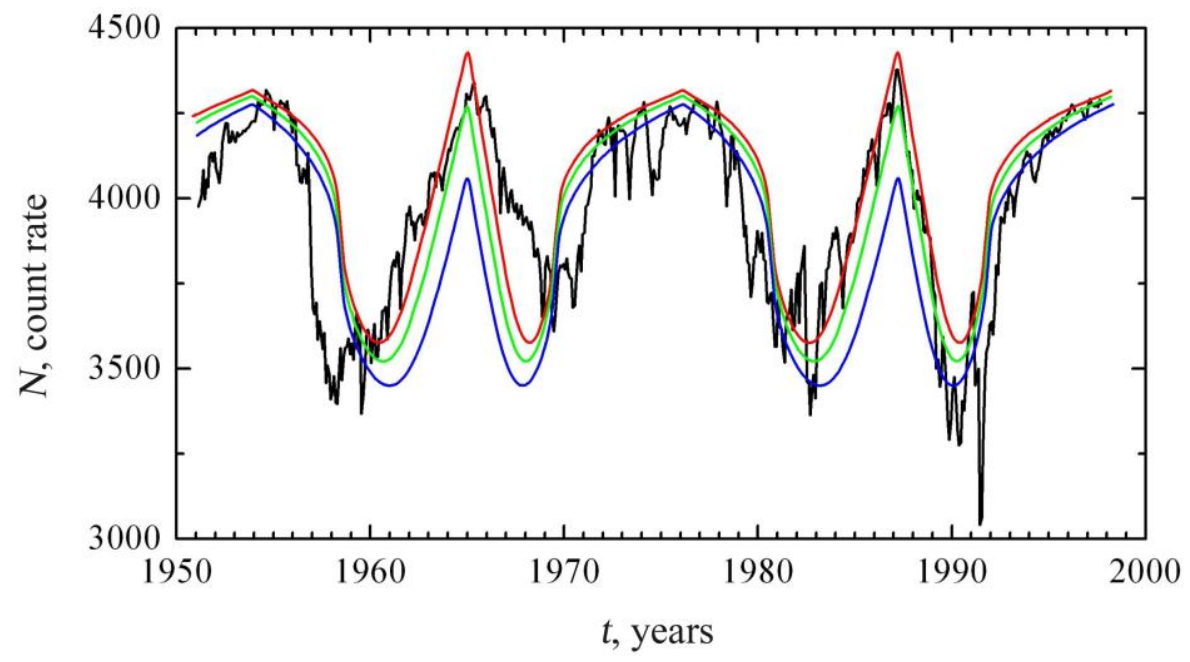

Figure 1. Time dependence of CR intensity as deduced from measurements made by the neutron monitor of the Climax station (black curve). Blue, green, and red curves are expected intensities for values of the ratio of regular and turbulent IMF intensities, and $k_{0}=3,5$, and 10 respectively 
As can be seen from the figure, with a large level of residual turbulence $\left(k_{0} \gtrsim 3\right)$, the sharp peak is strongly suppressed.

The comparison of these calculations with observations shows the overall correspondence between the observed and calculated intensities both in curve shape and in magnitude.

It should be emphasized that the theory has no adjustable parameters, and the only variable parameter best fits the observed data for $k_{0} \simeq 5$.

Figure 2 presents observed data on CR intensity inferred from measurements in the stratosphere over Moscow and Murmansk [Krainev et al., 2013]. The model predictions for these observations obtained in the above approximation are shown in the figure by solid and dotted curves.

The calculations are based on the same residual level of turbulence $k_{0}=5$ as in the high-energy region. Figure 2 indicates that despite the above simplifications, for SA cycles 19-23 there is in general satisfactory agreement between the theory and experiment. For them, the theory correctly describes the absolute value of CR intensity and its variations. However, it is clear that the situation is different for the observed CR behavior in SA cycle 23. This fact is discussed below.
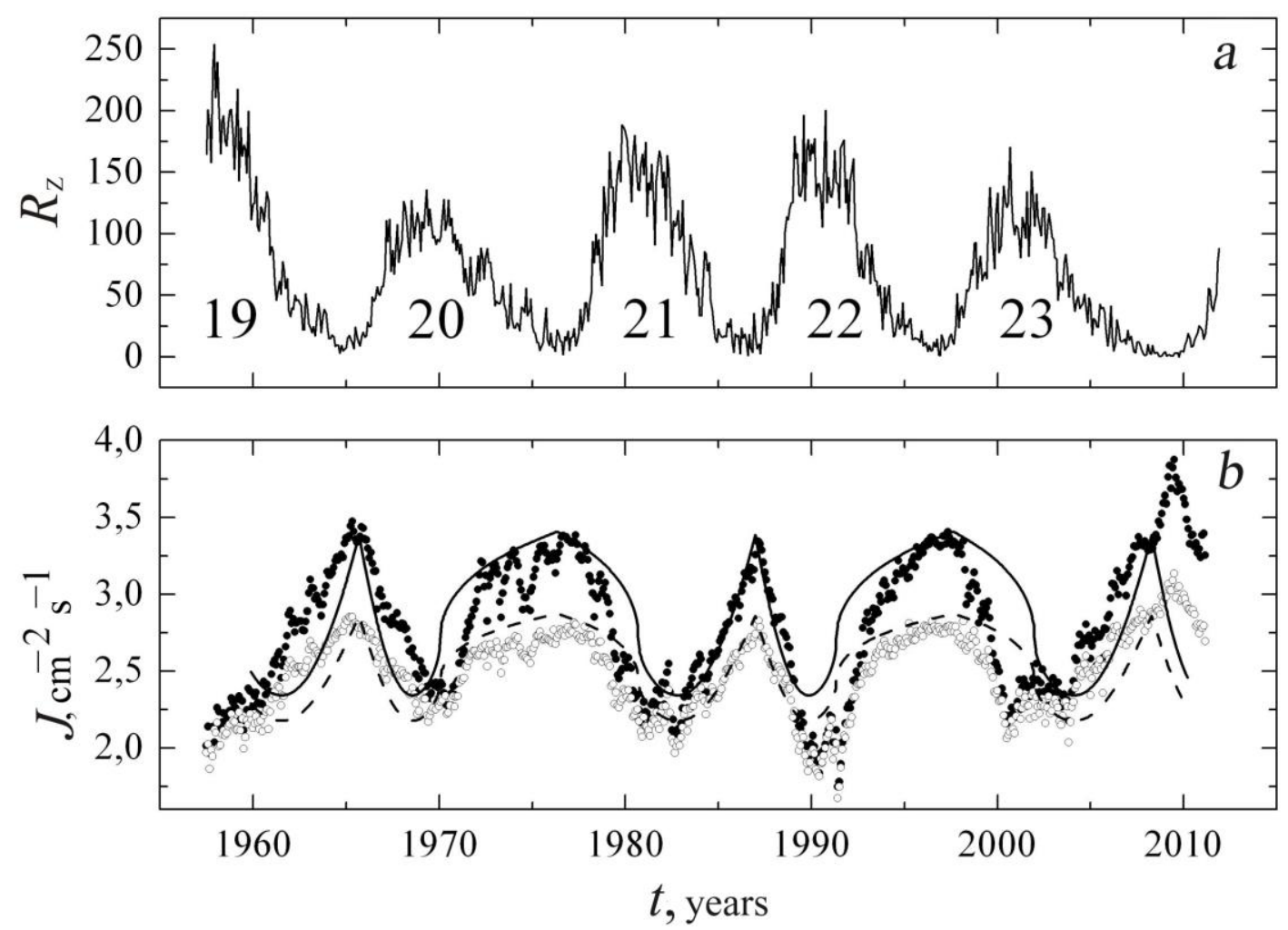

Figure 2. Time dependence of the sunspot number $(a)$, long-term CR flux variations $J$ as deduced from stratospheric measurements at stations Murmansk $\left(R_{\mathrm{c}}=0.6 \mathrm{GV}\right.$, solid circles $)$ and Moscow $\left(R_{\mathrm{c}}=2.4 \mathrm{GV}\right.$, open circles $)$ ( $b$ ). Model calculations for $k_{0}=5$ (dotted curve) and $k_{0}=15$ (solid curve) 
To describe the behavior of $\mathrm{CR}$ with even lower energies $\left(E_{\mathrm{p}} \sim 100 \mathrm{MeV}\right)$, we consider the mechanism which controls the behavior of regular and turbulent fields. In this case, to minimize changes in the theory, we consider the radial component of the regular field in the northern and southern hemispheres to be homogeneous, independent of heliolatitude and longitude, average the ratio of these fields over a sphere and refer it to the entire heliosphere.

The parameter $k$ obtained from these averages determines the modulation of high-energy CR coming to Earth from outside from two channels: from high latitudes and along the low-latitude layer. According to [Krymsky et al., 2007], the CR distribution function undergoes corresponding variations with SA cycle.

The comparison of the CR intensity registered by the spacecraft IMP- 8 with the model calculation is illustrated in Figure 3. The analysis shows that there is satisfactory agreement for $145-440 \mathrm{MeV}$ protons.

\section{COMPARISON WITH OBSERVATIONAL RESULTS RECEIVED IN YAKUTSK}

We analyze the period 1980-2012 - from the maximum of cycle 21 to early cycle 24 . The analysis is based on monthly averages of $\mathrm{CR}$ intensity recorded in Yakutsk with the neutron monitor and vertical muon telescope, mounted at $0 \mathrm{~m}$ of water equivalent (w.e.) [Krymsky et al., 2013]. These instruments form the integral part of the Yakutsk CR spectrograph after A.I. Kuzmin and are characterized by effective energies of particle detection of 13 and $32.5 \mathrm{GeV}$ respectively. More detailed information on the $\mathrm{CR}$ spectrograph is available on the website [http://hecrlab.ysn.ru].

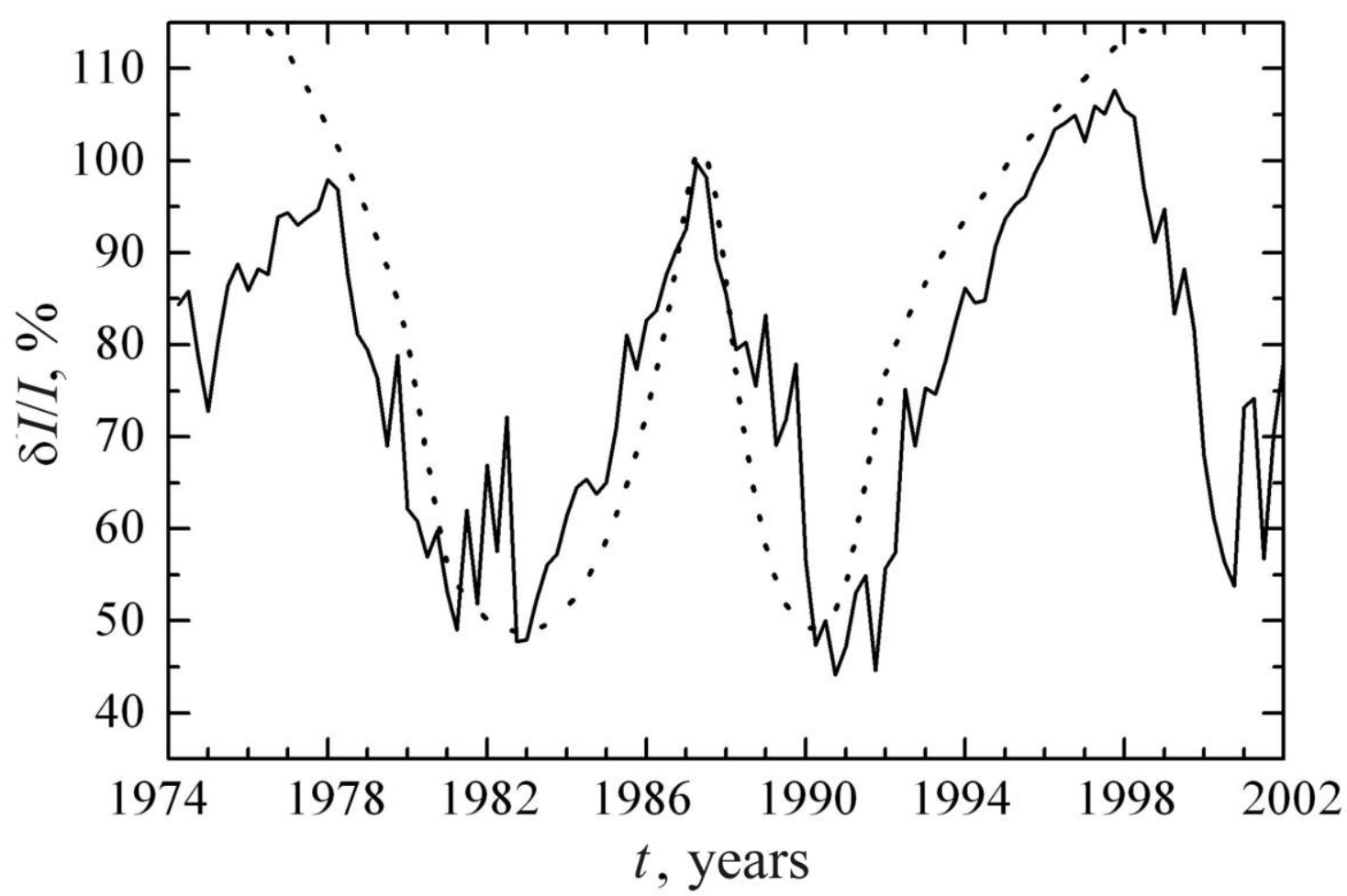

Figure 3. Time dependence of CR Intensity $\delta I / I$ inferred from proton measurements at the spacecraft IMP- 8 through differential channels $145-440 \mathrm{MeV}$. The dotted line corresponds to the model calculations with $k_{0}=5$ 
Results of multi-year CR-intensity measurements in Yakutsk are presented in Figure 4. It is apparent that for a long time CR intensity maxima in various solar cycles had almost the same values. But in the deep SA minimum between solar cycles 23 and 24 in 2008-2010, the Yakutsk spectrograph recorded an unusual and significant excess of CR intensity compared to all previous maxima. According to data from the neutron monitor, this excess amounts to about $5 \%$ (Figure 4, $a$ ); from the muon telescope, to about $3 \%$ (Figure 4, b). As mentioned above, the same picture is demonstrated by other authors.

\section{MECHANISM OF TURBULENT-FIELD GENERATION}

The given basic model in general satisfactorily describes the modulation observed in the neutron CR component [Krymsky et al., 2007], stratospheric measurements [Stozhkov et al., 2009; Krainev et al., 2013], and in the muon intensity [Krymsky et al., 2013].
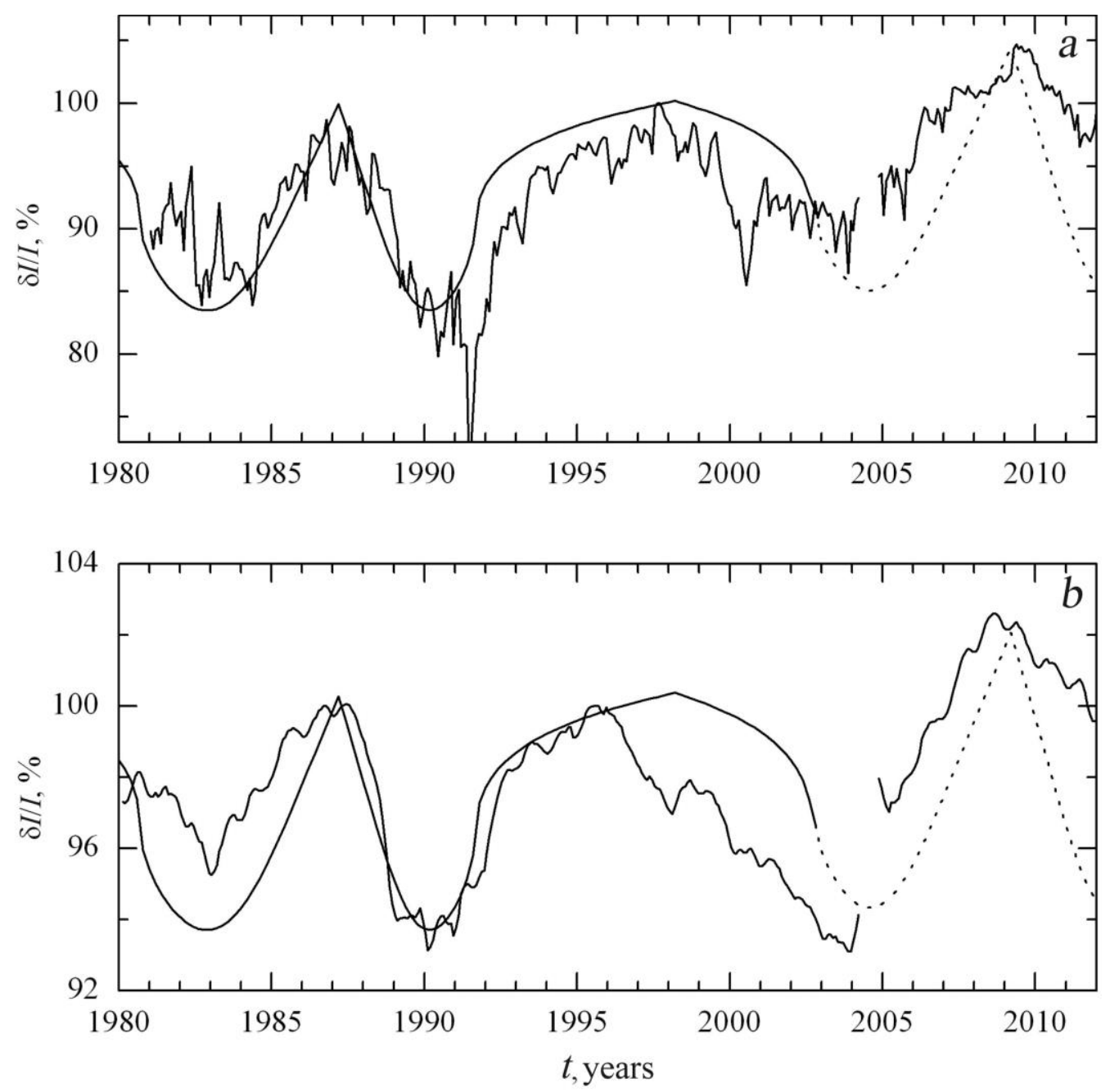

Figure 4. Time dependence of CR intensity $\delta I / I$ as deduced from measurements at the Yakutsk spectrograph: the neutron monitor $a$; the muon telescope mounted at $0 \mathrm{~m}$ of water equivalent and detecting particles coming from the vertical direction $b$. Solid and dotted curves represent model calculations for values of free parameter $k_{0}=5$ and $k_{0}=15$ respectively 
However, the varying ratio of the regular field to the turbulent one, entered into the theory, is a phenomenological parameter, whose behavior is not associated with any physical mechanism. Here we examine the possible mechanism which controls the behavior of the regular and turbulent fields. At the same time, to minimize changes in the theory, we continue considering the radial component of the regular field in the northern and southern hemispheres as homogeneous, independent of heliolatitude and longitude, average the ratio of these fields over a sphere and refer it to the entire heliosphere.

The parameter $k$, obtained from these averages, determines the modulation of high-energy CR coming to Earth through two channels: from high latitudes and along the low-latitude layer. The joint action of the high-latitude and low-latitude modulation channels produces a variation

$$
F(k)=b_{1} F(k) .
$$

A key element in the basic model of modulation is the level of magnetic turbulence. The fundamental assumption we adopt is that turbulence in SW occurs as a result of the interaction between fast and slow SW.

Idealize the problem setting. Suppose that the fast wind from high-latitude solar regions covers most of the northern and southern hemispheres, while the slow wind is located in the low-latitude layer and occupies the solid angle much smaller than $4 \pi$. This layer coincides with the Sun's magnetic equator and during a magnetic cycle deviates from the solar equatorial plane; the deviation becomes maximum at maximum activity when the layer passes through both poles of the Sun.

The curve describing "somersault" of the solar magnetic dipole in the magnetic cycle and demonstrating a change in the polar angle of its axis [Bravo, Stewart, 1995] has the simplest form if drawn in "deformed time". The period of the solar cycle decrease far exceeds that of increase. To make the cycle parameters symmetric, we add the deformed time $t_{\mathrm{sh}}(t)$ such that the cycle maximum falls exactly into the middle of the cycle:

$$
t_{\mathrm{sh}}(t)=\left[\sqrt{\left[\frac{4 T_{\mathrm{s}}-T}{4\left(T-2 T_{\mathrm{s}}\right)}\right]^{2}+\frac{t}{2\left(T-2 T_{\mathrm{s}}\right)}}-\frac{4 T_{\mathrm{s}}-T}{4\left(T-2 T_{\mathrm{s}}\right)}\right] T .
$$

Here $T$ is the cycle period, $T_{\mathrm{s}}$ is the period of SA increase. At $t=\left(0, T_{\mathrm{s}}, T\right)$, the formula yields $t_{\mathrm{sh}}=(0, T / 2, T)$.

From here on, we refer most calculations to the deformed time. The polar angle of the magnetic pole is set equal to $\theta_{0}$. Parametrize the somersault of the magnetic dipole as

$$
\theta_{0}\left(t_{\mathrm{sh}}\right)=\pi\left\{\begin{array}{cc}
\left(2 t_{\mathrm{sh}} / T\right)^{\alpha} / 2 & , 0<t_{\mathrm{sh}}<T / 2 \\
1-\left[2\left(T-t_{\mathrm{sh}}\right) / T\right]^{\alpha} / 2 & , T / 2<t_{\mathrm{sh}}<T
\end{array}\right.
$$

Here $\alpha=0.3$. If each time we take the pole that is located in the northern hemisphere, we have $0<\theta_{0}<\pi / 2$.

Address the interaction between fast and slow winds with $u_{+}$and $u_{-}$velocities in one of the hemispheres, say, in the northern one. For simplicity, the boundary between fast and slow winds - contact surface - is placed in the magnetic equatorial plane, and the equator is considered tilted by $\theta_{0}$. Then, near the Sun the contact surface is described by the equation 


$$
\cos \varphi=\operatorname{ctg} \theta_{0} \operatorname{ctg} \theta
$$

The longitudinal angle $\varphi$ is measured from the meridian, opposite to that on which the axis of the magnetic dipole lies. With distance away from the Sun, the contact surface is curved and is described by the equation

$$
\cos \left(\varphi-\frac{\omega_{\odot} r}{u}\right)=\operatorname{ctg} \theta_{0} \operatorname{ctg} \theta
$$

Here, $u=\left(u_{+}+u_{-}\right) / 2$ is the average SW velocity, and $\omega_{\odot}$ is the angular rotational velocity of the Sun.

Compute the angle $\beta$ between the normal to the contact surface and the radius vector $\vec{r}$. If the plane forms angles $\alpha_{1}, \alpha_{2}$ with the axes $\mathrm{X}, \mathrm{Y}$, then with the XY plane it makes an angle $\alpha$ and

$$
\operatorname{tg}^{2} \alpha=\operatorname{tg}^{2} \alpha_{1}+\operatorname{tg}^{2} \alpha_{2}
$$

Therefore, for the angle $\beta$ we have

$$
\operatorname{tg} \beta=\sqrt{\left(\frac{1}{r} \frac{\partial r}{\partial \theta}\right)^{2}+\left(\frac{1}{r \sin \theta} \frac{\partial r}{\partial \varphi}\right)^{2}}
$$

Counting the derivatives, we find

$$
\operatorname{tg}^{2} \beta=-\left(\frac{u}{\omega_{\odot} r}\right)^{2} \frac{2}{\cos 2 \theta_{0}+\cos 2 \theta}
$$

The denominator on the right-hand side is always less than zero, so the expression is positive.

The smaller is the angle $\beta$, the stronger is the collision between fast and slow winds. The flow velocity normal to the surface

$$
\Delta u=\cos \beta \frac{u_{+}-u_{-}}{2}
$$

If it is higher than the Alfvén velocity $v_{\mathrm{A}}=H / \sqrt{4 \pi \rho_{0}}$, a shock wave arises. The energy released in the shock wave first forms turbulence and then converts to heat. This is the basic assumption upon which we evaluate the intensity of the turbulent field. Accordingly, we assume that half of the gas pressure behind the shock front is provided by the turbulent magnetic field. Equations for mass balance, pressure, and energy density

$$
\begin{aligned}
& \rho \Delta u_{1}=\rho_{0} \Delta u, \\
& \rho \Delta u_{1}^{2}+P_{*}=\rho_{0} \Delta u^{2}, \\
& \frac{1}{2} \rho \Delta u_{1}^{3}+\frac{S_{*}}{S_{*}-1} P_{*} \Delta u_{1}=\frac{1}{2} \rho_{0} \Delta u^{3}
\end{aligned}
$$


are written in terms of the effective pressure $P_{*}$ and the effective adiabatic exponent $s *$ which are defined by equalities

$$
\begin{aligned}
& P_{*}=P+\frac{H^{2}}{8 \pi}\left[\left(\frac{\rho}{\rho_{0}}\right)^{2}-1\right], \\
& \frac{s_{*}}{S_{*}-1} P_{*}=\frac{5}{2} P+2 \frac{H^{2}}{8 \pi}\left[\left(\frac{\rho}{\rho_{0}}\right)^{2}-\frac{\Delta u}{\Delta u_{1}}\right] .
\end{aligned}
$$

Extra terms on the right-hand sides of these equations represent respectively the pressure of the regular magnetic field, which is parallel to the front, and the magnetic energy flux. The solution of the balance equations is

$$
\begin{aligned}
& \Delta u_{1}=\frac{s_{*}-1}{s_{*}+1} \nabla u, \\
& P_{*}=\frac{2}{s_{*}+1} \rho_{0} \Delta u^{2} .
\end{aligned}
$$

Next, we add a yet unknown compression ratio $\sigma=\left(s_{*}+1\right) /\left(s_{*-1}\right)$, insert $P_{*}$ in respective equations, and exclude $P$ from them; then we find

$$
\sigma=\sqrt{M_{\mathrm{A}}^{4}+13 M_{\mathrm{A}}^{2}+25 / 4}-\left(M_{\mathrm{A}}^{2}+5 / 2\right)
$$

Here

$$
M_{\mathrm{A}}^{2}=\frac{4 \pi \rho_{0} \Delta u^{2}}{H^{2}}>1
$$

is the squared magnetic Mach number. This number depends on $H, \rho_{0}$, and $\beta$. The shock wave appears where it is greater than 1.

The gas pressure

$$
P=\rho_{0} \Delta u^{2}\left[\frac{1}{\sigma}-\frac{\sigma+1}{2 M_{\mathrm{A}}^{2}}\right](\sigma-1) .
$$

Here $\rho_{0}=\rho_{\oplus}(R / r)^{2} ; \rho_{\oplus}=m_{\mathrm{p}} n_{\oplus} ; n_{\oplus}=8 \mathrm{~cm}^{-3}, m_{\mathrm{p}}$ is the proton mass.

The turbulent field strength

$$
H_{\mathrm{T}}=\sqrt{12 \pi P},
$$

because its adiabatic exponent equals $4 / 3$.

The regular field strength is proportional to $H_{\oplus}=5 \cdot 10^{-5}$ Oe:

$$
H=H_{\oplus} \sqrt{\frac{R_{\oplus}^{2}}{r^{2}}+\left(\frac{\omega R_{\oplus}}{u}\right)^{2} \sin ^{2} \theta} .
$$


The basic theory uses the parameter $k=H / H_{\mathrm{T}}$, which can now be calculated as a function of $\theta, \varphi$. In the directions without shock wave, this parameter becomes infinite, but this does not impede the calculation of the averaged $k$ because the harmonic averaging is applied, i.e. averaging of inverse value. Thus, the value of $k^{-1}$ is integrated over the sphere and is referred to $4 \pi$. The result depends on the tilt angle of the solar magnetic dipole $\theta_{0}$. The method for deriving effective $k$ ignores yet another aspect. The magnetic flux coming from the Sun to northern and southern hemispheres varies with the angle $\theta_{0}$ as in both a field of opposite sign emerges. Therefore, the average field $H$ must be multiplied by $\left(1-2 \theta_{0} / \pi\right)$. We see that during solar cycle maximum when $\theta=\pi / 2$, the effective regular field disappears. In view of the foregoing, we should apply corrections for $k$ and $p_{1}$, included in $b_{0}$. Both these values should be multiplied by the above factor. During minimum solar activity when $\theta_{0}=0$, the parameter $k$ becomes infinite due to the absence of a turbulence source. The basic model postulates the existence of residual turbulence, whereby the parameter $k$ is limited and according to observational data $k_{\max }=5$. We leave this assumption valid for our case as well.

Thus, for each instant of time during the magnetic cycle, we can calculate the deformed time $\tau$, solar dipole tilt $\theta_{0}$, the parameter $k$, and hence the modulation magnitude $f / f_{0}$.

The respective calculation for $p=13 \mathrm{GeV} / \mathrm{s}$ is shown in Figure 5. Data from the neutron monitor in Oulu [Gerasimova et al., 2015] show correspondence with the calculation in both magnitude and curve shape. Accordingly, the previously postulated behavior of the turbulent and regular fields in a solar cycle is justified by a particular mechanism of turbulence generation.

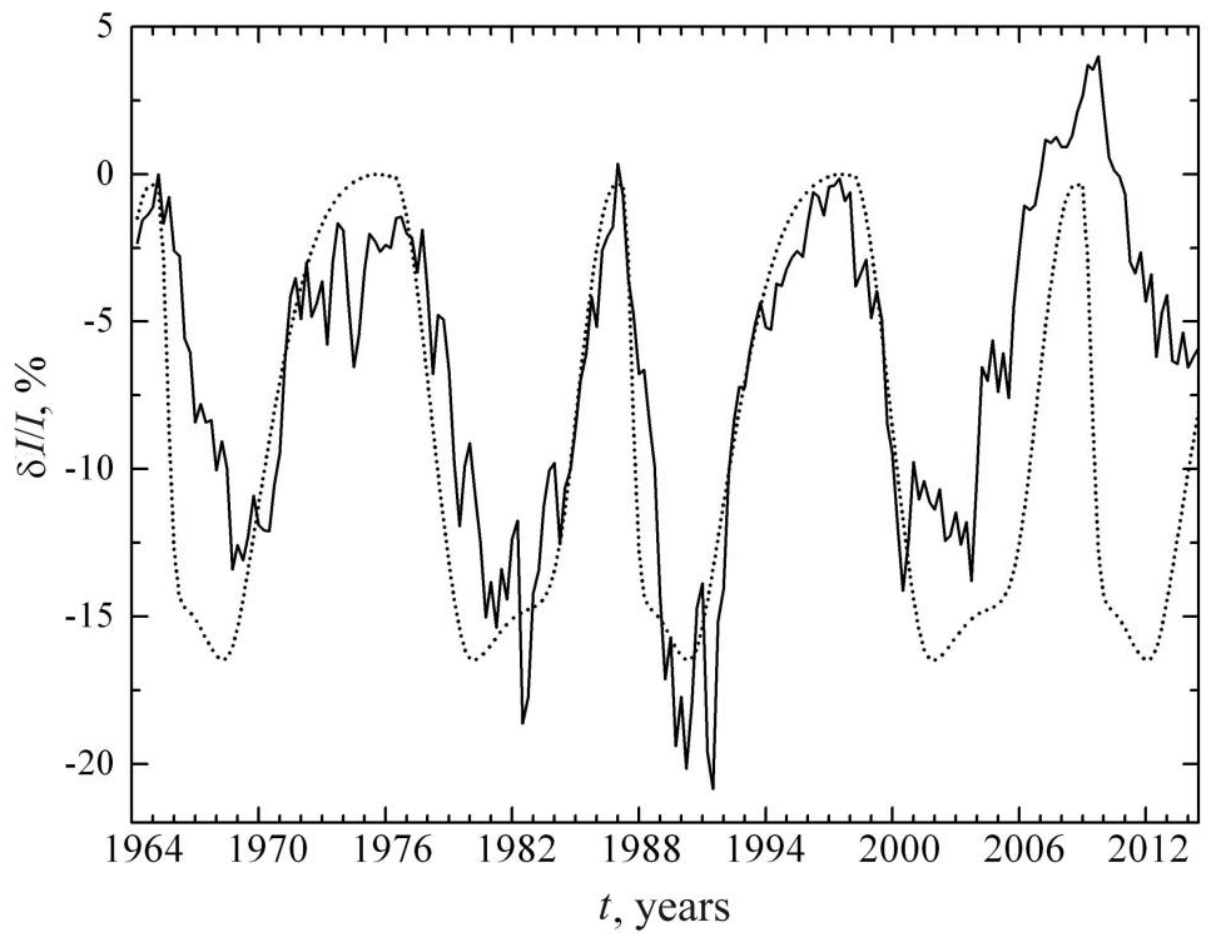

Figure 5. Time dependence of CR intensity as deduced from Oulu neutron monitor measurements. The dotted curve indicates the model calculations with $k_{0}=5$ 


\section{ANOMALY OF SOLAR CYCLE 23}

As noted above, at the end of SA cycle 23, the high-energy CR intensity, when the sharp peak remained unchanged, significantly exceeded all previously observed values. Referring to Figure 2, the agreement between the theory and observations was suddenly broken in SA cycle 23. According to data from the network of neutron monitors, the CR intensity in December 2009 peaked, exceeding its values obtained in cycles 19-22 by 5-6\%. Cosmic ray balloon and probe-ball measurements in the stratosphere showed a yet greater excess (> $20 \%)$ [Stozhkov et al., 2009]. However, many authors believe that the cause of this anomaly is an unusually long period of solar minimum and previously unobserved minimum value of IMF.

Cycle 23 exhibits a number of distinctive features. Grigoryev and Starodubtsev [Starodubtsev, Grigoryev, 2011] have studied changes in energy spectra of Forbush decreases in SA cycles 20-23. They revealed that the index of spectral tilt of Forbush decreases in cycle 23 is more rigid than that in the previous three cycles. This suggests that in SA cycle 23 the mirror mechanism played a key role in the formation of CR intensity decreases. To be effective, the mechanism also requires the presence of a sufficiently laminar magnetic field in the interplanetary space [Krymsky et al., 2009]. Furthermore, the authors have also established that entire cycle 23 as compared to previous ones is characterized by a reduced level of SW turbulence in the energetic region of frequency spectrum. The same fact is associated with the low CR anisotropy observed throughout SA cycle 23 [Gerasimova et al., 2011]. Thus, we have good reason to believe that throughout SA cycle 23 there was a more regular IMF as compared to the previous three cycles.

In this case, the unusual behavior of the CR intensity can be related to a decrease in the degree of scattering in resonant interaction between CR flux and SW inhomogeneities having spatial scales of $\sim 10^{12} \mathrm{~cm}$. This indicates that for SA cycle 23 we should take a much greater value of the free model parameter $k_{0}$. Indeed, the calculations show that the formal agreement between the theory and experiment in cycle 23 is reached when $k_{0}=15$.

It is still early to finally conclude that the agreement between the theory and experiment is restored.

\section{NECESSITY OF MODIFYING THE MODEL}

Our attention is engaged by the peculiarity of the model related to the level of residual turbulence, which is characterized by $k_{0}$. Aside from solar cycle 23, this parameter is surprisingly stable: in all cycles it is equal to 5 and remains this value for all CR energy ranges considered. Its value is determined by the requirement that maximum CR intensities during minima of even and odd cycles coincide, as inferred from observations. The theoretical value for positive and negative periods is given by expressions (when $k^{2}>>1$ )

$$
\begin{aligned}
& f^{+}=-b_{1} / 2, \\
& f^{-}=b_{1}\left(1 / 2-\lambda / k_{0}\right) .
\end{aligned}
$$

Hence it follows that $k_{0}=\lambda=4.6$. This coincidence of $k_{0}$ indicates a disadvantage of the model. The analysis of initial positions leads to the conclusion that we should abandon the assumption that the heliosphere is electrically neutral. 
The assumption about electrical neutrality entails a certain way of destroying the magnetic flux carried out by SW: half of the solar flux dissipates in the solar equatorial plane, and the other half is concentrated in poles and dissipates there. Hence it follows that the solar equatorial plane has a nonzero electric potential, which changes sign under reversal. The need to compensate the influence of the potential on the modulation leads to the requirement $k_{0}=4.6$.

If the assumption about the electrical neutrality is replaced by the assumption about zero electric potential of the equatorial plane, all model predictions are still valid, but in this case $k_{0}>>\lambda$. The above turbulence generation mechanism requires that during minimum SA $k_{0} \rightarrow \infty$.

What, then, is the mechanism for destruction of the magnetic flux? This mechanism is most likely to be the interstellar-gas flow past the heliosphere. If the gas velocity at infinity is parallel to the solar equatorial plane, the electric potential in this plane is zero. As shown in [Kurt, Mironova 2013], the interstellar-gas flow past is really close to that which we need (the ecliptic latitude of the point of downstream direction is $-5^{\circ}$ ).

\section{CAUSE OF THE ANOMALOUS CR INTENSITY INCREASE IN CYCLE 23}

The modified model of the heliosphere with zero potential is no longer capable of describing the abnormal increase through a decrease in the level of turbulence in SW. Here we study the modulating effect of the subsonic wind outside a standing shock wave, following mainly the findings of [Krimsky, Krivoshapkin, 2002]. As shown in that work, CR liberate a power of $\sim 2 \mathrm{GW}$ in Earth's atmosphere. Modulation reduces this value. For minimum SA we should assume that the supersonic SW does not contain an appreciable turbulent field and does not have a modulating effect. However, the subsonic wind behind a standing shock wave front should carry a turbulent field and continue to modulate CR. This modulation can be calculated supposing that the shock wave is spherical and the flow behind the front is incompressible. Let us estimate the diffusion coefficient, taking for it the Bohm value in the turbulent field $H_{\mathrm{T}}$, and find the field itself, assuming that a third of wind energy is expended for its generation. Then

$$
H_{\mathrm{T}}=\sqrt{3 \pi \rho_{\oplus}} u_{0} \frac{R_{\oplus}}{R}
$$

where $\rho_{\oplus}$ is the SW density in Earth's orbit, $R_{\oplus}$ is the orbital radius, $R$ is the shock wave radius,$u_{0}$ is the supersonic wind velocity.

Solving the particle transport equation in the region behind the shock front, we find that their intensity decreases due to modulation by a factor $\exp \left(-p_{\mathrm{t}} / p\right)$, where

$$
p_{\mathrm{t}}=\frac{3 u_{0}^{2}}{4 c^{2}} e R_{\oplus} \sqrt{3 \pi \rho_{\oplus}} .
$$

Substituting here the typical values $u_{0}=4 \cdot 10^{7} \mathrm{~cm} / \mathrm{s}, \rho_{\oplus}=8 \cdot 1.7 \cdot 10^{-24} \mathrm{~g} / \mathrm{cm}^{3}$, we have $p_{\mathrm{t}}=2.4 \mathrm{GeV} / \mathrm{s}$. This value corresponds to the 20 percent reduction in power emitted by CR. An increase in CR intensity to approximately the same value (according to radiocarbon data) occurred during the Maunder minimum. Obviously, the same mechanism is responsible for the CR intensity increase in cycle 23. 


\section{CONCLUSION}

The studies carried out allow us to make the following conclusions.

1. The description of the heliospheric CR modulation using the model minimized in terms of the number of parameters can be considered successful. The heliospheric model contains regular and turbulent magnetic fields the ratio of which exhibits sawlike variations with SA cycle.

2. We consider the interaction between regions of fast and slow SW and the formation of shock waves as a possible mechanism for generation of a turbulent magnetic field. Properties of CR modulation as a consequence of this mechanism can well reproduce observable 22-year variations.

3. The only time-dependent parameter of the model does not depend on the energy of particles. This indicates the power law spectrum of turbulence with an exponent close to -1 .

4. The initial assumption about electrical neutrality of the heliosphere appeared to be wrong. The zero potential needed to match the model with observations in the solar equatorial plane can be achieved if the frontal point, which is flowed around by interstellar gas of the heliosphere, lies near the plane.

5. The abnormal increase in CR intensity at the end of solar cycle 23 has the same nature as increases during the Maunder minimum and other similar cycles. These increases are associated with the residual modulation produced by the subsonic SW behind the standing shock wave front. During anomalies, the given modulation disappears.

This work was partially supported by grants of the Russian Foundation for Basic Research №15-42-05085r_vostok_a, №15-42-05083-r_vostok_a, and by the RAS Presidium Program № 23.

\section{REFERENCES}

Belov A.V., Shelting B.D., Gushchina R.T., Obridko V.N., Kharshiladze A.F., Yanke V.G. Global magnetic field of the Sun and long-term vatiations of galactic cosmic rays. J. Atmos. Solar-Terr. Phys. 2001, vol. 63, pp. 1923-1929. DOI: 10.1016/S13646826(01)00073-6.

Belov A.V., Gushchina R.T., Obridko V.N., Shelting B.D., Yanke V.G. Connection of the long-term modulation of cosmic rays with the parameters of the global magnetic field of the Sun. Geomagn. Aeron. 2002, vol. 42, pp. 693-700.

Belov A.V., Gushchina R.T., Obridko V.N., Shelting B.D., Yanke V.G. The relation of the global magnetic solar field indices and the solar wind characteristics with the long-term variations of galactic cosmic rays. Proceeding of the 29th ICRC. Pune. 2005, vol. 2, pp. 235-238.

Belov A.V., Gushchina R.T., Obridko V.N., Shel'ting B.D., Yanke V.G. Simulation of the modulation of galactic cosmic rays during solar activity cycles 21-23. Bull. Russ. Acad. Sci.: Phys. 2007, vol. 71, pp. 974-976. DOI: 10.3103/S1062873807070246.

Bisoi S.K., Janardhan P., Ingale M., Subramanian P., Ananthakrishnan S., Tokumaru M., Fujiki K. A study of dens ity modulation index in the inner helioshperic solar wind during solar cycle 23. Astrophys. J. 2014, vol. 795, pp. 69-76. DOI: $10.1088 / 0004-637 \mathrm{X} / 795 / 1 / 69$. 
Bravo S., Stewart G. The inclination of the heliomagnetic equator and the presence of an inclined relic field in the Sun. Astrophys. J. 1995, vol. 446, pp. 431-434. DOI 10.1086/ 175801.

Charakhch'yan A.N., Stozhkov Y.I., Svirzhevsky N.S., Charakhch'yan T.N. Anomalous effect in the 11 -year galactic cosmic ray modulation. Proceeding of the 13th ICRC. Denver. 1973, vol. 2, pp. 1159-1164.

Ferreira S.E.S., Potgieter M.S. Long-term cosmic-ray modulation in the heliosphere. Astrophys. J. 2004, vol. 603, pp. 744752. DOI: $10.1086 / 381649$.

Gerasimova S.K., Gololobov P.Yu., Grigoryev V.G., Krivoshapkin P.A., Krymsky G.F., Starodubtsev S.A. Heliospheric modulation of cosmic rays in the 23rd solar cycle and in previous cycles. Proceeding of the 32nd ICRC. Beijing. 2011, vol. 11, pp.180-183.

Gerasimova S.K., Krymsky G.F., Krivoshapkin P.A., Gololobov P.Y., Starodubtsev S.A. A modified basic model of the heliospheric modulation of cosmic rays. Bull. Russ. Acad. Sci.: Phys. 2015, vol. 79, pp. 613-615. DOI: 10.3103/ S1062873815050214.

Gushchina R.T., Belov A.V., Obridko V.N., Shelting B.D. Manifestations of cyclic variations in the solar magnetic field in long-term modulation of cosmic ray. Geomagn. Aeron. 2008, vol. 48, pp. 571-577. DOI: 10.1134/S0016793208050022.

Gushchina R.T., Belov A.V., Eroshenko E.A., Obridko V.N., Paouris E., Shelting B.D. Cosmic ray modulation during the solar activity growth phase of cycle 24. Geomag. Aeron. 2014, vol. 54, pp. 430-436. DOI: 10.1134/S0016793214040057.

Hale G.E., Nicholson S.B.: The law of sun-spot polarity. Astrophys. J. 1925, vol. 62, pp. 270-300.

Jokipii J.R., Levy E.H. Electric field effects on galactic cosmic rays at the heliosphere boundary. Proc. 16th Internat. Cosmic Ray Conf. Kyoto. 1979, vol. 3, pp. 52-56.

Jokipii J.R., Kota J. Galactic and anomalous cosmic rays in the heliosphere. Invited, rapporteur and highlight papers, 25th ICRC. Durban. 1997, vol. 8, pp. 151-174.

Jokipii J.R., Thomas B. Effects of drift on the transport of cosmic rays. IV. Modulation by a wavy interplanetary current sheet. Astrophys. J. 1981, vol. 243, pp. 1115-1122. DOI: 10.1086/158675.

Jokipii J.R., Levy E.H., Hubbard W.B. Effects of particle drift on cosmic-ray transport. I. General properties, application to solar modulation. Astrophys. J. 1977, vol. 213, pp. 861-868. DOI: 10.1086/155218.

Kota J., Jokipii J.R. Effects of drift on the transport of cosmic rays. VI. A three dimensional model including diffusion. Astrophys. J. 1983, vol. 265, pp. 573-581. DOI: 10.1086/160701.

Krajnev M.B. The solar corona expansion geometry and cosmic ray effects. IV. On the cosmic ray energy change due to the electric field. Proc. 16th Internat. Cosmic Ray Conf. Kyoto. 1979, vol. 3, pp. 236-241.

Krainev M.B., Kalinin M.S. Arguments in support of influence of external electric field of heliosphere on galactic cosmic rays. Bull. Russ. Acad. Sci.: Phys. 2003, vol. 67, pp. 1439-1442.

Krainev M.B., Bazilevskaya G.A., Gerasimova S.K., Krivoshapkin P.A., Krymsky G.F., Starodubtsev S.A., Stozhkov Y.I., Svirzhevsky N.S. On the status of the sunspot and magnetic cycles in the galactic cosmic ray intensity. J. Phys .: Conf. Ser. 2013, vol. 409, 012,016. DOI: 10.1088/1742-6596/409/1/012016.

Krymsky G.F. Diffusion mechanism of diurnal variation of galactic cosmic rays. Geomagn. Aeron. 1964, vol. 4, pp. 763-769.

Krymsky G.F., Krivoshapkin P.A.: 2002, Cosmic rays in the past. Geomagn. Aeron. 2002, vol. 42, pp. 305-308.

Krymsky G.F., Krivoshapkin P.A., Mamrukova V.P., Gerasimova S.K. Heliospheric modulation of high-energy cosmic ray. I. Basic model of cosmic-ray modulation with solar cycle. J. Exp. Theor. Phys. 2007, vol. 104, pp. 189-195. DOI: 10.1134/ S1063776107020033.

Krymsky G.F., Krivoshapkin P.A., Mamrukova V.P., Gerasimova S.K. Piston shock and Forbush effect. Astronomy Letters. 2009, vol. 35, pp. 696-700. DOI: 10.1134/ S1063773709100065.

Krymsky, G.F. Gololobov, P.Y. Krivoshapkin P.A., Gerasimova S.K., Grigoryev V.G., Starodubtsev, S.A. Variation of the cosmic ray intensity in an 11-yr solar activity cycle: Experiment and theory. JETP Lett. 2013, vol. 98, pp. 769-772. DOI: 10.1134/ S0021364013250139.

Kurt V.G., Mironova E.N. Methods of local interstellar medium investigation. Phys. Usp. 2013, vol. 56, pp. 910918. DOI: 10.3367/UFNe.0183.201309e.0963. 
Laurenza M., Vecchio A., Storini M., Carbone V. Drift effects on the galactic cosmic ray modulation. Astrophys. J. 2014, vol. 781, pp. 71-82. DOI: 10.1088/0004-637X/781/2/71.

Levy E.H. Theory of solar magnetic cycle wave in diurnal-variation of energetic cosmic rays: Physical basis of anisotropy. J. Geophys. Res. 1976, vol. 81, pp. 2082-2088. DOI: 10.1029/JA081i013p02082.

Lockwood M. Solar change and climate: an update in the light of the current exceptional solar minimum. Proc. R. Soc.: A. 2010, vol. 466, pp. 303-329. DOI: 10.1098/rspa.2009.0519.

Lockwood J.A., Webber W.R. The 11-year solar modulation of cosmic rays as deduced from neutron monitor variations and direct measurements at low energies. J. Geophys. Res. 1967, vol. 72, pp. 5977-5989. DOI: 10.1029/ JZ072i023p05977.

Manuel R., Ferreira S.E.S., Potgieter M.S.Time-dependent modulation of cosmic rays in the heliosphere. Solar Phys. 2014, vol. 289, pp. 2207-2231. DOI: 10.1007/s11207-013-0445-y.

Manuel R., Ferreira S.E.S., Potgieter M.S., Strauss R.D., Engelbrecht N.E. Time-dependent cosmic rays modulation. Adv. Space Res. 2011, vol. 47, pp. 1529-1537. DOI: 10.1016/j.asr.2010.12.007.

Moraal H. Cosmic-ray modulation equations. Space Sci. Rev. 2013, vol. 176, pp. 299-319. DOI: 10.1007/s11214-0119819-3.

Pacini A.A., Usoskin I.G. An unusual pattern of cosmic-ray modulation during solar cycles 23 and 24. Solar Phys. 2015, vol. 290, pp. 943-950. DOI: 10.1007/s11207-014-0645-0.

Parker E.N. The passage of energetic charged particles through interplanetary space. Planet. Space Sci. 1965, vol. 13, pp. 9-49. DOI 10.1016/0032-0633(65)90131-5.

Potgieter M.S., Burger R.A., Ferreira S.E.S. Modulation of cosmic rays in the heliosphere from solar minimum to maximum: a theoretical perspective. Space Sci. Rev. 2001, vol. 97, pp. 295-307. DOI: 10.1023/A:1011837303094.

Starodubtsev S.A., Grigoryev V.G. Cosmic rays and solar wind turbulence: peculiarities of the 23rd solar cycle. Geomagn. Aeron. 2011, vol. 51, pp. 1004-1009. DOI: 10.1134/ S001679321107022X.

Stozhkov Y.I., Svirzhevsky N.S., Bazilevskaya G.A., Kvashnin A.N., Makhmutov V.S., Svirzhevskaya A.K. Long-term (50 years) measurements of cosmic ray fluxes in the atmosphere. Adv. Space Res. 2009, vol. 44, pp. 1124-1137. DOI: 10.1016/ j.asr.2008.10.038

Thomas S.R., Owens M.J., Lockwood M. The 22-year Hale cycle in cosmic ray flux: evidence for direct heliospheric modulation. Solar Phys. 2014, vol. 289, pp. 407-421. DOI: 10.1007/s11207-013-0341-5.

Wibberenz G., Cane H.V., Richardson I.G., von Rosenvinge T.T. The influence of tilt angle and magnetic field variations on cosmic ray modulation. Space Sci. Rev. 2001, vol. 97, pp. 343-347. DOI: 10.1023/A:1011849605820.

Zhao L.-L., Qin G., Zhang M., Heber B. Modulation of galactic cosmic rays during the unusual solar minimum between cycles 23 and 24. J. Geophys. Res. 2014, vol. 119, pp. 1493-1506. DOI: 10.1002/2013JA019550.

http://hecrlab.ysn.ru 